\title{
Myocardial strain characteristics and outcomes after transcatheter aortic valve replacement
}

\author{
Dominik Buckert, Raid Tibi, Maciej Cieslik, Michael Radermacher, Yang-Yang Qu, \\ Volker Rasche, Peter Bernhardt, Vinzenz Hombach, Wolfgang Rottbauer, Jochen Wöhrle \\ Department of Internal Medicine II, University of Ulm, Ulm, Germany
}

\begin{abstract}
Background: Objective of this study was to make an assessment of standard functional and deformation parameters (strain) in patients after transcatheter aortic valve replacement (TAVR) by cardiac magnetic resonance imaging (CMR) and the evaluation of their prognostic impact.

Methods: Patients undergoing TAVR received CMR on a 1.5 T whole-body scanner at 3 months after the procedure. Deformation parameters (strain, strain rate, velocity, displacement) were assessed in longitudinal, circumferential and radial orientation using a feature tracking approach. Primary outcome measure was defined according to Valve Academic Research Consortium-2 (VARC-2) criteria.

Results: Eighty-three patients formed the study population. Deformation parameters were significantly reduced in all three orientations for strain (longitudinal: $-12.1 \pm 5.4 \%$ vs. $-15.9 \pm 1.96 \%, p<0.0001$; radial: $34.4 \pm 15.3 \%$ vs. $47.2 \pm 11.4 \%, p<0.0001$; circumferential: $-16.8 \pm 4.3 \%$ vs. $-21.1 \pm 2.5 \%$, $p<0.0001$ ) and strain rate (longitudinal: $-0.79 \pm 0.33 \% / \mathrm{s} v$ s. $-0.91 \pm 0.23 \% / \mathrm{s}, p=0.043 ;$ radial: $2.5 \pm 1.2 \% / s$ vs. $2.9 \pm 0.9 \%, p=0.067$; circumferential: $-1.1 \pm 0.6 \% / s$ vs. $-1.3 \pm 0.3 \% / s, p=0.006$ ) in comparison to a healthy control population. Median follow-up was 614 days. During this period, 13 endpoints occurred (cumulative event rate of 10.7\%). Patients with event by trend exhibited poorer strain and strain rate in longitudinal and radial orientation without reaching statistical significance (longitudinal strain: $-11.2 \pm 5.4 \%$ vs. $-12.3 \pm 5.4 \%, p=0.52$; longitudinal strain rate: $-0.73 \pm$ $\pm 0.23 \% / s$ vs. $0.80 \pm 0.35 \% / s, p=0.53$; radial strain: $29.5 \pm 19.6 \%$ vs. $35.2 \pm 14.5 \%, p=0.24$; radial strain rate: $2.2 \pm 1.6 \% / s$ vs. $2.6 \pm 1.2 \% / s, p=0.31$ ).
\end{abstract}

Conclusions: Assessment of left ventricular deformation parameters by CMR revealed functional abnormalities in comparison to healthy controls. Prognostic significance remains to be further investigated. (Cardiol J 2018; 25, 2: 203-212)

Key words: cardiac magnetic resonance imaging, strain imaging, cardiac mechanics, severe aortic stenosis, transcatheter aortic valve replacement, outcomes and prognosis

\section{Introduction}

Aortic stenosis (AS) is the most common valvular pathology in western populations, especially in the elderly [1]. Indication for aortic valve replacement in patients with severe AS is given as soon as typical symptoms occur [2]. In patients at intermediate to high surgical risk, transcatheter aortic valve replacement (TAVR) has been shown to be superior to surgical valve replacement [3-5].

Several factors such as need for hemodynamic support during procedure, extent of paravalvular leakage, chronic kidney or pulmonary disease have been identified to influence early and midterm prognosis after TAVR $[6,7]$. However most

Address for correspondence: Dominik Buckert, MD, Department of Internal Medicine II, University Hospital Ulm, Albert-Einstein-Allee 23, 89081 Ulm, Germany, tel: +49 731500 45000, fax: +49 731500 45005, e-mail: dominik.buckert@uniklinik-ulm.de

Received: 27.07.2017 Accepted: 29.09.2017 
studies forming the evidence base concentrate on acute outcomes. Hence there is little data on outcomes beyond the first year of follow-up $[8,9]$. Valid prognostic information is of special interest as it might provide important contributions to risk stratification and clinical decision making especially in the context of present ongoing randomized trials in patients with a low risk for surgical valve replacement.

Cardiac magnetic resonance imaging (CMR) plays an increasing role in the diagnostic management of patients suffering from valvular pathologies [10]. In addition to its favorable advantages like high spatial and temporal resolution, lack of ionizing radiation and excellent reproducibility, it is considered the gold standard for the evaluation and quantification of left and right ventricular functional parameters such as volumes and ejection fractions [11]. Consequently, CMR has proven to provide important prognostic information in various valvular and non-valvular cardiac diseases [12-14]. Most recently, new parameters for the quantification of left ventricular mechanics and function have been introduced to CMR. The assessment of global and regional strain and subsequent parameters such as strain rate, displacement and velocity by feature tracking (FT) CMR is thought to have the potential to improve the quantification of cardiac function and thus might lead to an improvement of CMR's prognostic value [15-19].

The objective of this study was to make an assessment of standard functional and innovative deformation parameters in patients after TAVR by $\mathrm{CMR}$ and the evaluation of their prognostic impact.

\section{Methods}

\section{Study population}

Patients with symptomatic severe AS undergoing TAVR were enrolled between 2014 and 2015. Indication for valve replacement was given according to current guidelines [20]. All patients were considered eligible unless they exhibited predefined exclusion criteria such as cardiac or respiratory instability, metal implants or devices unsuitable for CMR, concomitant limiting disease, allergy against gadolinium based contrast agents or severely impaired renal function. Age and sex were matched with a healthy control population and was derived from a database formed within another project which was conducted for establishment of reference values. For these subjects, any form of relevant valvular or coronary artery disease, hypertrophic or dilative cardiomyopathy, diastolic dysfunction or inflammatory heart disease were ruled out.

The study was approved by the institutional ethics committee (clinicaltrials.gov: NCT02162069). Written informed consent was obtained from every patient as well as from healthy controls.

\section{CMR examination}

All patients received CMR examination at 3 months after TAVR. CMR imaging was performed on a $1.5 \mathrm{~T}$ whole-body clinical magnetic resonance scanner (Achieva 1.5T, Philips Medical Systems, Best, Netherlands) using a 32-channel phased-array receiver coil. CMR examinations were carried out in concordance with current guidelines [21,22]. A steady-state free precession sequence (SSFP, repetition time $3.4 \mathrm{~ms}$, echo time $1.7 \mathrm{~ms}$, voxel size $1.6 \times 1.6 \mathrm{~mm}$, flip-angle $\alpha 55^{\circ}$, slice thickness $8 \mathrm{~mm}$, acquisition in end-expiratory breath-hold, 32 cardiac phases) were used for functional imaging of the left and right ventricle in long and short axis orientation. CMR examinations and FT analyzes were performed identically for AS patients and healthy controls.

\section{CMR analysis}

Two experienced readers performed offline image analysis in a consensus approach. Epi- and endocardial contours were drawn manually in the long- and short-axis oriented SSFP-images. Basic functional (left and right ventricular ejection fraction [LVEF, RVEF], left and right ventricular end-diastolic volume index [LVEDI, RVEDVI], left ventricular mass/volume index [LVMVI], leftventricular mass divided by left-ventricular enddiastolic volume) and strain parameters (strain, systolic strain rate, displacement, systolic velocity) were derived from the SSFP cine images using the dedicated software $\mathrm{cvi}^{42 \circledast}$ (Version 5.2, Circle Cardiovascular Imaging, Calgary, Canada). Strain parameters in longitudinal and radial orientation were assessed globally and on a segmental basis for the left ventricle according to current recommendations [23]. Global circumferential function was derived from a mid-ventricular slice and expressed as positive number in case of a clockwise rotation (seen from the apex) or as a negative number in case of a counterclockwise movement (example depicted in Figure 1).

\section{Follow-up}

Patients had a follow-up after 3, 6, 12 and 24 months after TAVR. Primary outcome measure was defined according to the Valve Academic 


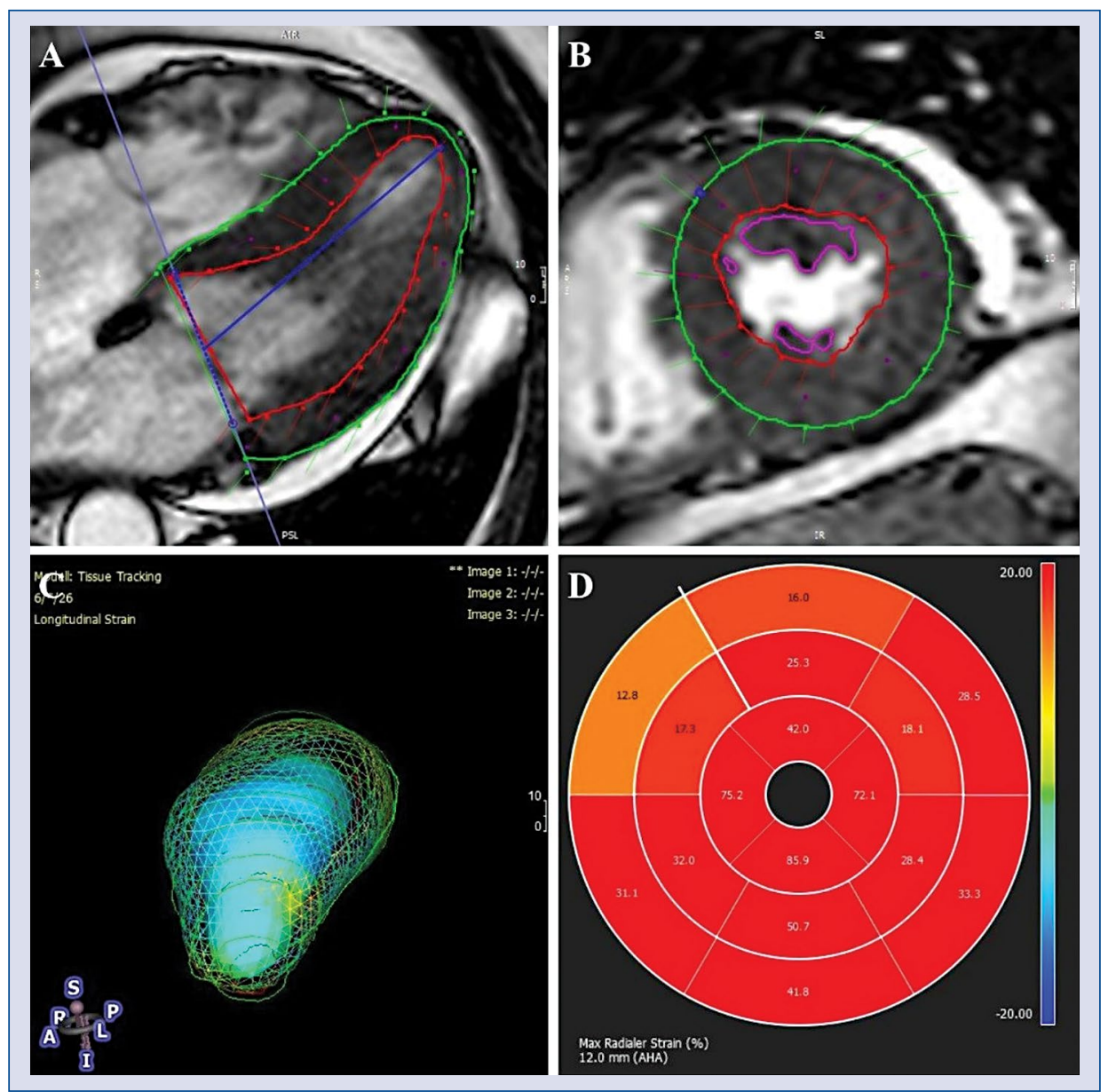

Figure 1. Example of deformation parameter assessment. Endocardial (red lining) and epicardial (green lining) in long axis (A) and short axis (B) orientation. Movement and deformation of strain vectors can be seen. A three-dimensional model is calculated based on two-dimensional information $(\mathbf{C})$ and results are depicted in a 16-segments bulls-eye plot (here for radial strain, D).

Research Consortium-2 (VARC-2) criteria. The composite endpoint death of any cause, non-fatal myocardial infarction, hospitalization due to heart failure and cerebral ischemia (not associated to index procedure, after hospital discharge) [24] was analyzed. Non-fatal myocardial infarction was defined according to the current universal definition [25].

\section{Statistical analysis}

To test the correlation between two categorical classification factors, the $\chi^{2}$ test was applied. Continuous variables were tested for normal distribution by the D'Agostino-Pearson test. Variables with normal distribution were reported as mean \pm standard deviation and a two-tailed t-test (for independent samples) was used for comparison. Variables without normal distribution were reported as median with percentiles and compared by the Mann-Whitney-U rank sum test. Univari- ate regression analyses using Cox's proportional hazard models were performed to estimate the predictive value of the variables. Hazard ratio (HR) and corresponding 95\% confidence interval (CI) are provided. A p-value $<0.05$ was considered significant. Statistical analyses were performed using commercially available software (Stata 13, College Station, USA, MedCalc, Mariakerke, Belgium).

\section{Results}

\section{Study population and functional} characteristics before TAVR

Eighty-three patients after TAVR for treatment of symptomatic severe AS were enrolled into the study. Mean age was $79.3 \pm 5.6$ years. Forty-three patients were men (51.8\%). Most of the patients presented in functional New York Heart Association (NYHA) class III or IV ( $\mathrm{n}=72$, 
Table 1. Baseline characteristics.

\begin{tabular}{lc}
\hline Variable & N $=83$ \\
\hline Age [years] & $79.3 \pm 5.6$ \\
Body mass index $\left[\mathrm{kg} / \mathrm{m}^{2}\right]$ & $27.7 \pm 4.7$ \\
Male & $43(51.8 \%)$ \\
Diabetes mellitus & $26(31.3 \%)$ \\
Arterial hypertension & $78(94.0 \%)$ \\
NYHA functional class (baseline): & \\
II & $11(13.2 \%)$ \\
III-IV & $72(86.8 \%)$ \\
Frailty & $43(51.8 \%)$ \\
STS score [\%] - mortality & $5.5 \pm 3.7$ \\
EuroSCORE II $\%$ ] & $5.0 \pm 3.6$ \\
Atrial fibrillation & $29(34.9 \%)$ \\
Coronary artery disease & $49(59.0 \%)$ \\
Cerebrovascular disease & $7(8.4 \%)$ \\
Chronic obstructive pulmonary disease & $9(10.8 \%)$ \\
eGFR > 60 mL/min & $48(57.8 \%)$ \\
Functional characteristics before TAVR & \\
LVEF [\%] & $57.6 \pm 15.7$ \\
Aortic valve area [cm ${ }^{2}$ ] & $0.76 \pm 0.24$ \\
Max PG [mm Hg] & $64.9 \pm 22.7$ \\
\hline
\end{tabular}

Values are mean \pm standard deviation or $\mathrm{n}(\%)$; eGFR - estimated glomerular filtration rate; LVEF — left ventricular ejection fraction; Max PG - maximal pressure gradient; NYHA - New York Heart Association; STS - Society of Thoracic Surgeons; TAVR - transcatheter aortic valve replacement

86.8\%). Mean EuroSCORE II was $5.0 \pm 3.6 \%$ [26], Society of Thoracic Surgeons (STS) score for mortality was $5.5 \pm 3.7 \%$ [27]. Table 1 depicts clinical baseline characteristics including relevant pre-existing conditions and functional characteristics before TAVR in more detail.

Mean LVEF was $57.6 \pm 15.7 \%$ before TAVR in echocardiography. Ten patients exhibited a LVEF $<35 \%$ (12.0\%) and 43 (51.8\%) patients had pulmonary hypertension. More than moderate mitral and tricuspid valve regurgitation was found in 18 $(22.5 \%)$ and $13(16.4 \%)$ cases, respectively.

\section{CMR assessment of basic functional and}

left ventricular deformation parameters

Three months after TAVR, LVEF as assessed by CMR was a mean $61.1 \pm 14.0 \%$. A severely impaired LVEF (LVEF < 35\%) was present in $5(6.0 \%)$ patients. In comparison to the control group with a mean LVEF of $68.4 \pm 5.4 \%$, the LVEF in the TAVR population was significantly lower $(\mathrm{p}=0.002)$. LVEDVI was significantly higher in the TAVR cohort $\left(78.9 \pm 23.2 \mathrm{~mL} / \mathrm{m}^{2}\right.$ vs. $65.2 \pm$ $\left.\pm 16.5 \mathrm{~mL} / \mathrm{m}^{2}, \mathrm{p}=0.001\right)$ as well as LVMVI
$(0.86 \pm 0.28 \mathrm{~g} / \mathrm{mL}$ vs. $0.70 \pm 0.08 \mathrm{~g} / \mathrm{mL}, \mathrm{p}=0.001)$ Right ventricular parameters did not statistically differ as detailed in Table 2.

Deformation analysis by FT CMR yielded significantly reduced results in all three orientations for strain (longitudinal: $-12.1 \pm 5.4 \%$ vs. $-15.9 \pm$ $\pm 1.96 \%, \mathrm{p}<0.0001$; radial: $34.4 \pm 15.3 \%$ vs. $47.2 \pm$ $\pm 11.4 \%, \mathrm{p}<0.0001$; circumferential: $-16.8 \pm 4.3 \%$ vs. $-21.1 \pm 2.5 \%, \mathrm{p}<0.0001)$. In addition the strain rate in the TAVR population was significantly reduced compared to the control group (longitudinal: $-0.79 \pm 0.33 \% / \mathrm{s}$ vs. $-0.91 \pm 0.23 \% / \mathrm{s}, \mathrm{p}=0.043$; radial: $2.5 \pm 1.2 \% / \mathrm{s}$ vs. $2.9 \pm 0.9 \%, \mathrm{p}=0.067$; circumferential: $-1.1 \pm 0.6 \% / \mathrm{s}$ vs. $-1.3 \pm 0.3 \% / \mathrm{s}$, $\mathrm{p}=0.006)$. Global peak displacement and global peak velocity in radial orientation were also significantly reduced in the TAVR population compared with the control population (displacement: $5.1 \pm$ $\pm 2.0 \mathrm{~mm}$ vs. $6.8 \pm 0.87 \mathrm{~mm}, \mathrm{p}<0.0001$; velocity: $33.1 \pm 11.9 \mathrm{~mm} / \mathrm{s}$ vs. $40.7 \pm 8.6 \mathrm{~mm} / \mathrm{s}, \mathrm{p}<0.001$ ). In addition, direction of global circumferential strain (clockwise vs. counterclockwise) significantly differed between the TAVR cohort and the control group ( $2.9 \pm 5.2$ deg vs. $-0.5 \pm 5.4 \mathrm{deg})$.

\section{Follow-up}

Median follow-up was 614 days with 61 (73.5\%) patients having 12 months follow-up. Thirty-five $(42.2 \%)$ patients had a follow-up period of more than 2 years. During the follow-up period, 13 endpoints occurred. Five (6.0\%) patients died. Two patients had non-fatal myocardial infarctions $(2.4 \%)$ and $3(3.6 \%)$ patients had to be hospitalized because of heart failure. Cerebral ischemia occurred in $2(2.4 \%)$ subjects. Cumulative event rate was $10.7 \%$.

\section{Predictors of outcomes}

Table 3 depicts characteristics of patients with vs. without event. There is no significant difference with regard to basic clinical features such as age, sex, diabetes or hypertension. Established risk prediction parameters (NYHA class at 3 months follow-up, frailty, STS score and EuroSCORE II) also showed comparable results for both groups. The frequency of cardiac and non-cardiac comorbidities (presence of coronary artery disease, chronic obstructive lung disease, pulmonary hypertension, severe mitral or tricuspid valve regurgitation, paravalvular leakage $>1$ ) was also not significantly different. Patients with event exhibited poorer left and right ventricular functional parameters without reaching statistical significance (LVEF: $57.3 \pm$ $\pm 11.4 \%$ vs. $61.7 \pm 14.4 \%, \mathrm{p}=0.32$; LVEDVI: 
Table 2. Comparison of functional and deformation parameters of study cohort after transcatheter aortic valve replacement (TAVR) and control group.

\begin{tabular}{|c|c|c|c|}
\hline Variable & TAVR cohort $(n=83$ ) & Control group $(n=39)$ & $\mathbf{P}$ \\
\hline LVEF [\%] & $61.1 \pm 14.0$ & $68.4 \pm 5.4$ & $<0.002$ \\
\hline LVEDVI [mL/m²] & $78.9 \pm 23.2$ & $65.2 \pm 16.5$ & $<0.001$ \\
\hline LVMVI $[\mathrm{g} / \mathrm{mL}]$ & $0.86 \pm 0.28$ & $0.70 \pm 0.08$ & $<0.001$ \\
\hline RVEF [\%] & $61.7 \pm 12.6$ & $65.4 \pm 8.0$ & 0.10 \\
\hline RVEF $<35$ & $2(2.4 \%)$ & $0(0 \%)$ & 0.09 \\
\hline RVEDVI [mL/m²] & $69.7 \pm 17.3$ & $66.1 \pm 17.7$ & 0.29 \\
\hline \multicolumn{4}{|l|}{ Global longitudinal: } \\
\hline Peak strain [\%] & $-12.1 \pm 5.4$ & $-15.9 \pm 1.96$ & $<0.0001$ \\
\hline Peak strain rate [\%/s] & $-0.79 \pm 0.33$ & $-0.91 \pm 0.23$ & 0.043 \\
\hline Peak displacement [mm] & $3.9 \pm 2.1$ & $3.3 \pm 1.3$ & 0.10 \\
\hline Peak velocity $[\mathrm{mm} / \mathrm{s}]$ & $39.8 \pm 16.5$ & $42.0 \pm 12.4$ & 0.46 \\
\hline \multicolumn{4}{|l|}{ Global radial: } \\
\hline Peak strain [\%] & $34.4 \pm 15.3$ & $47.2 \pm 11.4$ & $<0.0001$ \\
\hline Peak strain rate $[\% / s]$ & $2.5 \pm 1.2$ & $2.9 \pm 0.9$ & 0.07 \\
\hline Peak displacement [mm] & $5.1 \pm 2.0$ & $6.8 \pm 0.87$ & $<0.0001$ \\
\hline Peak velocity $[\mathrm{mm} / \mathrm{s}]$ & $33.1 \pm 11.9$ & $40.7 \pm 8.6$ & $<0.001$ \\
\hline \multicolumn{4}{|c|}{ Mid-ventricular circumferential: } \\
\hline Peak strain [\%] & $-16.8 \pm 4.3$ & $-21.1 \pm 2.5$ & $<0.0001$ \\
\hline Peak strain rate $[\% / s]$ & $-1.1 \pm 0.4$ & $-1.3 \pm 0.3$ & $<0.006$ \\
\hline Peak displacement [deg] & $2.9 \pm 5.2$ & $-0.5 \pm 5.4$ & $<0.001$ \\
\hline Peak velocity [deg/s] & $36.0 \pm 63.3$ & $24.4 \pm 70.2$ & 0.36 \\
\hline
\end{tabular}

Values are mean \pm standard deviation or $n(\%)$. LVEDVI — left ventricular end-diastolic volume index; LVEF — left ventricular ejection fraction; LVMVI — left ventricular mass/volume index; RVEDVI — right ventricular end-diastolic volume index; RVEF — right ventricular ejection fraction

$83.5 \pm 22.7 \mathrm{~mL} / \mathrm{m}^{2}$ vs. $78.1 \pm 23.3 \mathrm{~mL} / \mathrm{m}^{2}, \mathrm{p}=0.46$ LVMVI: $0.83 \pm 0.3 \mathrm{~g} / \mathrm{mL}$ vs. $0.86 \pm 0.3 \mathrm{~g} / \mathrm{mL}$, $\mathrm{p}=0.75 ;$ RVEF: $56.9 \pm 10.8 \%$ vs. $62.6 \pm 12.7 \%$, $\mathrm{p}=0.15$; RVEDVI: $74.6 \pm 24.8 \mathrm{~mL} / \mathrm{m}^{2}$ vs. $68.8 \pm$ $\pm 15.7 \mathrm{~mL} / \mathrm{m}^{2}, \mathrm{p}=0.28$ ). Peak strain and peak strain rate in longitudinal and radial orientation tended to be reduced, mid-ventricular circumferential strain did not differ (longitudinal strain: $-11.2 \pm$ $\pm 5.4 \%$ vs. $-12.3 \pm 5.4 \%, \mathrm{p}=0.52$; longitudinal strain rate: $-0.73 \pm 0.23 \% / \mathrm{s}$ vs. $0.80 \pm 0.35 \% / \mathrm{s}$, $\mathrm{p}=0.53$; radial strain: $29.5 \pm 19.6 \%$ vs. $35.2 \pm$ $\pm 14.5 \%, \mathrm{p}=0.24$; radial strain rate: $2.2 \pm 1.6 \% / \mathrm{s}$ vs. $2.6 \pm 1.2 \% / \mathrm{s}, \mathrm{p}=0.31$ ).

Table 4 compares patients after TAVR without endpoint and healthy controls. Global peak strain was reduced in all orientations (longitudinal: $-12.3 \pm 5.4 \%$ vs. $-15.9 \pm 2.0, \mathrm{p}<0.0001$; radial: $35.2 \pm 14.5 \%$ vs. $47.2 \pm 11.4 \%, \mathrm{p}<0.0001$; mid-ventricular circumferential: $-16.8 \pm 4.5 \%$ vs. $-21.1 \pm 2.5 \%, \mathrm{p}<0.0001)$.

In Table 5 , analyses of univariate predictors of endpoints are shown. NYHA functional class 3 months after TAVR was the only parameter with significant correlation to event-free survival. Patients persisting in NYHA class III or IV exhibited a HR of $6.69(\mathrm{p}=0.02)$. Clinical features, risk prediction models, comorbidities and CMR parameters were not significantly associated with the occurrence of endpoints.

\section{Discussion}

The present study evaluates cardiac deformation characteristics of patients 3 months after TAVR and analyzes their correlation to event-free survival in a 2 year follow-up. The main finding is that left-ventricular deformation parameters (strain, strain rate, displacement and velocity) in longitudinal, radial and circumferential orientation were significantly reduced 3 months after successful TAVR in comparison to a healthy control group. This was also true for patients with preserved ejection fraction. Patients with endpoint tended to exhibit lower strain values in comparison to patients without event, though statistical significance was not reached. Despite having higher values by 
Table 3. Comparison of patients with versus without event.

\begin{tabular}{|c|c|c|c|}
\hline Variable & Event $(n=12)$ & No event $(n=71)$ & $\mathbf{P}$ \\
\hline LVEF [\%] & $57.3 \pm 11.4$ & $61.7 \pm 14.4$ & 0.32 \\
\hline LVEDVI $\left[\mathrm{mL} / \mathrm{m}^{2}\right]$ & $83.5 \pm 22.7$ & $78.1 \pm 23.3$ & 0.46 \\
\hline LVMVI $[\mathrm{g} / \mathrm{mL}]$ & $0.83 \pm 0.3$ & $0.86 \pm 0.3$ & 0.75 \\
\hline RVEF [\%] & $56.9 \pm 10.8$ & $62.6 \pm 12.7$ & 0.15 \\
\hline RVEDVI $\left[\mathrm{mL} / \mathrm{m}^{2}\right]$ & $74.6 \pm 24.8$ & $68.8 \pm 15.7$ & 0.28 \\
\hline \multicolumn{4}{|l|}{ Global longitudinal: } \\
\hline Peak strain [\%] & $-11.2 \pm 5.4$ & $-12.3 \pm 5.4$ & 0.52 \\
\hline Peak strain rate $[\% / s]$ & $-0.74 \pm 0.23$ & $-0.80 \pm 0.35$ & 0.53 \\
\hline Peak displacement [mm] & $3.9 \pm 2.3$ & $3.9 \pm 2.1$ & 1.00 \\
\hline Peak velocity $[\mathrm{mm} / \mathrm{s}]$ & $38.7 \pm 17.8$ & $40.0 \pm 16.4$ & 0.80 \\
\hline \multicolumn{4}{|l|}{ Global radial: } \\
\hline Peak strain [\%] & $29.5 \pm 19.6$ & $35.2 \pm 14.5$ & 0.24 \\
\hline Peak strain rate $[\% / s]$ & $2.2 \pm 1.6$ & $2.6 \pm 1.2$ & 0.31 \\
\hline Peak displacement [mm] & $4.8 \pm 2.6$ & $5.2 \pm 1.9$ & 0.53 \\
\hline Peak velocity $[\mathrm{mm} / \mathrm{s}]$ & $34.0 \pm 9.6$ & $33.0 \pm 12.3$ & 0.79 \\
\hline \multicolumn{4}{|c|}{ Mid-ventricular circumferential: } \\
\hline Peak strain [\%] & $-16.8 \pm 2.7$ & $-16.8 \pm 4.5$ & 1.00 \\
\hline Peak strain rate $[\% / s]$ & $-1.1 \pm 0.4$ & $-1.1 \pm 0.3$ & 1.00 \\
\hline Peak displacement [deg] & $1.1 \pm 4.2$ & $3.2 \pm 5.3$ & 0.20 \\
\hline Peak velocity [deg/s] & $44.4 \pm 63.6$ & $34.5 \pm 64.3$ & 0.62 \\
\hline
\end{tabular}

Values are mean \pm standard deviation; LVEDVI — left ventricular end-diastolic volume index; LVEF — left ventricular ejection fraction;

LVMVI — left ventricular mass/volume index; RVEDVI — right ventricular end-diastolic volume index; RVEF — right ventricular ejection fraction

trend, deformation characteristics of AS patients without event still were significantly reduced in comparison to normal controls.

Strain imaging is an emerging technique for the non-invasive evaluation of global and regional left ventricular function [28]. Thus far, most studies addressing this topic are derived from two-dimensional speckle-tracking echocardiography. There are various studies proving prognostic significance of particular speckle-tracking derived strain parameters, foremost being global longitudinal strain. This parameter has proven to be significantly correlated to adverse events in several settings, from heart transplant patients to severe AS [29, 30]. Though this technique is highly accepted and widely available, it exhibits several inherent limitations such as poor inter-reader reproducibility and need for an appropriate 'acoustic window' [31, 32]. FT CMR offers the possibility to derive deformation parameters from standard cine sequences and thus combines the advantages of both imaging modalities $[15,16]$. CMR derived left-ventricular strain was an independent predictor of survival in patients with dilated cardiomyopathy [33]. Moreover, it proved to provide significant prognostic power concerning the occurrence of major adverse cardiac events in an all-comers CMR population [34].

The findings of the present study are in concordance with an earlier study using speckle tracking echocardiography for the assessment of cardiac mechanics in AS patients before and after surgical valve replacement [35]. Patients in this study exhibited significantly lower values for longitudinal $(-14.6 \pm 4.1 \%$ vs. $-20.3 \pm 2.3 \%$, p < 0.001$)$, radial $(33.1 \pm 14.8 \%$ vs. $38.9 \pm 6.4 \%, \mathrm{p}<0.001)$ and circumferential strain $(-15.2 \pm 5.0 \%$ vs. $-19.5 \pm 2.9 \%, \mathrm{p}<0.001$ ) before TAVR in comparison to normal controls, despite normal LVEF $(60 \pm 12 \%)$. It could be demonstrated that valve replacement lead to an improvement in cardiac function (longitudinal strain: from $-14.6 \pm 4.1 \%$ to $-16.4 \pm 4.5 \%, \mathrm{p}<0.001$; radial strain: from $33.1 \pm$ $\pm 14.8 \%$ to $37.2 \pm 13.4 \%, \mathrm{p}=0.02$; circumferential strain: from $-15.2 \pm 5.0 \%$ to $-18.0 \pm 5.3 \%$, $\mathrm{p}<0.001$ ) but that deformation parameters still were reduced in comparison to healthy controls, especially in longitudinal direction $(-16.9 \pm 4.8 \%$ vs. $-20.3 \pm 2.3 \%, \mathrm{p}<0.0001)$.

A recent study proved the strong correlation between CMR derived deformation parameters and 
Table 4. Comparison of patients without event and controls.

\begin{tabular}{|c|c|c|c|}
\hline Variable & No event $(n=71)$ & Control group $(n=39)$ & $\mathbf{P}$ \\
\hline LVEF [\%] & $61.7 \pm 14.4$ & $68.4 \pm 5.4$ & $<0.006$ \\
\hline LVEDVI $\left[\mathrm{mL} / \mathrm{m}^{2}\right]$ & $78.1 \pm 23.3$ & $65.2 \pm 16.5$ & $<0.003$ \\
\hline LVMVI [g/mL] & $0.86 \pm 0.3$ & $0.70 \pm 0.08$ & $<0.002$ \\
\hline RVEF [\%] & $62.6 \pm 12.7$ & $65.4 \pm 8.0$ & 0.22 \\
\hline RVEDVI $\left[\mathrm{mL} / \mathrm{m}^{2}\right]$ & $68.8 \pm 15.7$ & $66.1 \pm 17.7$ & 0.41 \\
\hline \multicolumn{4}{|l|}{ Global longitudinal: } \\
\hline Peak strain [\%] & $-12.3 \pm 5.4$ & $-15.9 \pm 2.0$ & $<0.0001$ \\
\hline Peak strain rate $[\% / s]$ & $-0.80 \pm 0.35$ & $-0.91 \pm 0.23$ & 0.08 \\
\hline Peak displacement [mm] & $3.9 \pm 2.1$ & $3.3 \pm 1.3$ & 0.11 \\
\hline Peak velocity $[\mathrm{mm} / \mathrm{s}]$ & $40.0 \pm 16.4$ & $42.0 \pm 12.4$ & 0.51 \\
\hline \multicolumn{4}{|l|}{ Global radial: } \\
\hline Peak strain [\%] & $35.2 \pm 14.5$ & $47.2 \pm 11.4$ & $<0.0001$ \\
\hline Peak strain rate $[\% / \mathrm{s}]$ & $2.6 \pm 1.2$ & $2.9 \pm 0.9$ & 0.18 \\
\hline Peak displacement [mm] & $5.2 \pm 1.9$ & $6.8 \pm 0.87$ & $<0.0001$ \\
\hline Peak velocity $[\mathrm{mm} / \mathrm{s}]$ & $33.0 \pm 12.3$ & $40.7 \pm 8.6$ & $<0.001$ \\
\hline \multicolumn{4}{|c|}{ Mid-ventricular circumferential: } \\
\hline Peak strain [\%] & $-16.8 \pm 4.5$ & $-21.1 \pm 2.5$ & $<0.0001$ \\
\hline Peak strain rate $[\% / s]$ & $-1.1 \pm 0.3$ & $-1.3 \pm 0.3$ & $<0.001$ \\
\hline Peak displacement [deg] & $3.2 \pm 5.3$ & $-0.5 \pm 5.4$ & $<0.001$ \\
\hline Peak velocity [deg/s] & $34.5 \pm 64.3$ & $24.4 \pm 70.2$ & 0.45 \\
\hline
\end{tabular}

Values are mean \pm standard deviation; LVEDVI — left ventricular end-diastolic volume index; LVEF — left ventricular ejection fraction; LVMVI — left ventricular mass/volume index; RVEDVI — right ventricular end-diastolic volume index; RVEF — right ventricular ejection fraction

the underlying hemodynamic AS subtype [36]. As it could be demonstrated that positive response after TAVR could be predicted by analysis of longitudinal strain and velocity, it has been suggested that the evaluation of cardiac mechanics could be useful for the right timing of valve replacement. Nevertheless, prognostic implication of persisting subtle functional abnormalities after TAVR remain poorly investigated and are thus unclear [37]. A correlation of deformation parameters to outcomes have not as yet been reported.

Many studies report that early events in the first few weeks after TAVR are mainly related to the procedure itself including major vascular complications, bleeding complications, cerebral infarction or severe paravalvular aortic insufficiency $[6,9]$. Our cohort consisted of patients with a follow-up CMR 3 months after successful aortic valve replacement. Therefore, early events within the first few weeks after TAVR were excluded from analyses. Though this approach might introduce a certain selection bias, it could be appropriate for the proper identification of predictors of mid- and long-term outcomes after interventional valve replacement. Hence, patients surviving 3 months after TAVR had an excellent prognosis and exhibited only relatively low event rates.

Several observational and register studies sought to identify factors predicting outcomes in patients after TAVR $[9,38,39]$. It is of note that most of these studies reported non-cardiac morbidities being causative for deaths and rehospitalizations during follow-up $>30$ days after valve replacement. Interestingly, in all of the studies addressing this issue, left ventricular functional parameters such as LVEF and LVEDVI did not have statistical impact on outcomes after valve replacement, indicating that non-cardiac conditions gain influence after successful treatment of AS. In concordance to this assumption, a strong correlation of NYHA functional class after TAVR and the occurrence of endpoints could be observed in the present study.

Nevertheless, since indication for TAVR is constantly expanding toward patients at low to intermediate risk, it can be assumed that functional cardiac parameters will show greater prognostic power in this population exhibiting lower frequencies of severe comorbidities [40]. Several studies have suggested that the evaluation of deformation characteristics might provide beneficial additive 
Table 5. Univariate predictors of composite endpoint.

\begin{tabular}{|c|c|c|c|}
\hline Variable & Hazard ratio & $95 \% \mathrm{Cl}$ & $\mathbf{P}$ \\
\hline Age [per year] & 1.01 & $0.91-1.12$ & 0.83 \\
\hline Body mass index [per $\left.\mathrm{kg} / \mathrm{m}^{2}\right]$ & 0.99 & $0.88-1.13$ & 0.93 \\
\hline Male & 3.36 & $0.90-12.47$ & 0.07 \\
\hline Diabetes mellitus & 0.65 & $0.18-2.42$ & 0.52 \\
\hline Arterial hypertension & 0.52 & $0.07-4.08$ & 0.53 \\
\hline NYHA functional class (follow-up) III-IV & 6.69 & $1.38-32.36$ & 0.02 \\
\hline Frailty & 0.88 & $0.28-2.84$ & 0.84 \\
\hline STS score $(\%)$ - mortality & 1.01 & $0.86-1.17$ & 0.95 \\
\hline EuroSCORE II [\%] & 1.05 & $0.91-1.21$ & 0.50 \\
\hline Atrial fibrillation & 1.31 & $0.39-4.35$ & 0.66 \\
\hline Coronary artery disease & 0.26 & $0.06-1.21$ & 0.09 \\
\hline Chronic obstructive pulmonary disease & 2.65 & $0.71-9.82$ & 0.15 \\
\hline \multicolumn{4}{|l|}{ Systolic pulmonary artery pressure: } \\
\hline $31-55 \mathrm{~mm} \mathrm{Hg}$ & 1.15 & $0.37-3.59$ & 0.81 \\
\hline$>55 \mathrm{~mm} \mathrm{Hg}$ & 1.27 & $0.33-4.85$ & 0.73 \\
\hline Paravalvular leak $>1$ & 1.12 & $0.33-3.85$ & 0.86 \\
\hline LVEF [\%] & 0.98 & $0.94-1.02$ & 0.29 \\
\hline LVEDVI $\left[\mathrm{mL} / \mathrm{m}^{2}\right]$ & 1.01 & $0.99-1.03$ & 0.37 \\
\hline LVMVI [g/mL] & 1.42 & $0.15-13.15$ & 0.76 \\
\hline RVEF [\%] & 0.98 & $0.95-1.01$ & 0.20 \\
\hline RVEDVI $\left[\mathrm{mL} / \mathrm{m}^{2}\right]$ & 1.01 & $0.98-1.05$ & 0.39 \\
\hline \multicolumn{4}{|l|}{ Global longitudinal: } \\
\hline Peak strain [\%] & 1.05 & $0.94-1.16$ & 0.41 \\
\hline Peak strain rate $[\% / s]$ & 0.99 & $0.97-1.01$ & 0.40 \\
\hline Peak displacement [mm] & 0.97 & $0.75-1.32$ & 0.97 \\
\hline Peak velocity $[\mathrm{mm} / \mathrm{s}]$ & 0.74 & $0.96-1.03$ & 0.74 \\
\hline \multicolumn{4}{|l|}{ Global radial: } \\
\hline Peak strain [\%] & 0.98 & $0.95-1.01$ & 0.22 \\
\hline Peak strain rate $[\% / s]$ & 1.00 & $0.99-1.00$ & 0.24 \\
\hline Peak displacement [mm] & 0.94 & $0.72-1.23$ & 0.67 \\
\hline Peak velocity $[\mathrm{mm} / \mathrm{s}]$ & 1.01 & $0.96-1.06$ & 0.82 \\
\hline \multicolumn{4}{|l|}{ Mid-ventricular circumferential: } \\
\hline Peak strain [\%] & 1.00 & $0.80-1.26$ & 0.98 \\
\hline Peak strain rate $[\% / s]$ & 0.94 & $0.08-10.96$ & 0.96 \\
\hline Peak displacement [deg] & 0.89 & $0.74-1.08$ & 0.24 \\
\hline Peak velocity [deg/s] & 1.00 & $0.98-1.02$ & 0.99 \\
\hline
\end{tabular}

$\mathrm{Cl}$ - confidence interval; LVEDVI - left ventricular end-diastolic volume index; LVEF — left ventricular ejection fraction; LVMVI - left ventricular mass/volume index; NYHA - New York Heart Association; RVEDVI — right ventricular end-diastolic volume index; RVEF — right ventricular ejection fraction; STS - Society of Thoracic Surgeons

information over the assessment of basic functional parameters alone [15, 16].

In the present study, a significant association of deformation parameters and poor outcome could not be proven. However, patients with endpoint had lower strain values by trend than patients without endpoint. It can be assumed that the investigated parameters will gain significance in larger AS cohorts and longer follow-up periods, particularly in low-risk AS populations. Thus, evaluation of deformation and mechanical characteristics by CMR seem to be a promising approach to improve understanding of cardiac functional properties and the prognostic implications linked to them. 


\section{Limitations of the study}

In this study, patients with severe AS were compared to healthy controls. Since AS is promoted by well-known risk factors like arterial hypertension and hyperlipoproteinemia that also might cause other cardiac morbidities (e.g. coronary artery disease), it is difficult to control for confounding systemic or cardiac conditions that might influence cardiac deformation parameters independently from an existing severe AS.

There is no consensus to date on how to assess and report strain parameters. Therefore, values reported in this study are to a large extent modality and software specific. Comparability between different vendors, imaging modalities and post-processing software needs to be further evaluated and proven.

\section{Conclusions}

Assessment of left ventricular deformation parameters by CMR revealed subtle functional abnormalities in comparison to healthy controls. Prognostic significance remains to be further investigated.

\section{Conflict of interest: None declared}

\section{References}

1. Coffey S, Cairns BJ, Iung B. The modern epidemiology of heart valve disease. Heart. 2016; 102(1): 75-85, doi: 10.1136/ heartjnl-2014-307020, indexed in Pubmed: 26541169.

2. Vahanian A, Alfieri O, Andreotti F, et al. ESC Committee for Practice Guidelines (CPG), Joint Task Force on the Management of Valvular Heart Disease of the European Society of Cardiology (ESC), European Association for Cardio-Thoracic Surgery (EACTS). Guidelines on the management of valvular heart disease (version 2012): the Joint Task Force on the Management of Valvular Heart Disease of the European Society of Cardiology (ESC) and the European Association for Cardio-Thoracic Surgery (EACTS). Eur J Cardiothorac Surg. 2012; 42(4): S1-44, doi: 10.1093/ejcts/ezs455, indexed in Pubmed: 22922698.

3. Leon MB, Smith CR, Mack MJ, et al. Transcatheter or Surgical Aortic-Valve Replacement in Intermediate-Risk Patients. N Engl J Med. 2016; 374: 1609-1620.

4. Adams DH, Popma JJ, Reardon MJ, et al. Transcatheter aorticvalve replacement with a self-expanding prosthesis. N Engl J Med. 2014; 370(19): 1790-1798, doi: 10.1056/NEJMoa1400590, indexed in Pubmed: 24678937.

5. Holmes DR, et al. Jr, Mack MJ, Kaul S 2012 ACCF/AATS/SCAI/ STS expert consensus document on transcatheter aortic valve replacement: developed in collabration with the American Heart Association, American Society of Echocardiography, European Association for Cardio-Thoracic Surgery, Heart Failure Society of America, Mended Hearts, Society of Cardiovascular Anesthesiologists, Society of Cardiovascular Computed Tomography, and Society for Cardiovascular Magnetic Resonance. J Thorac Cardiovasc Surg. 2012; 144: e29-84.

6. Tamburino C, Capodanno D, Ramondo A, et al. Incidence and predictors of early and late mortality after transcatheter aortic valve implantation in 663 patients with severe aortic stenosis. Circulation. 2011; 123(3): 299-308, doi: 10.1161/CIRCULATIONAHA.110.946533, indexed in Pubmed:21220731.

7. Thomas M, Schymik G, Walther T, et al. One-year outcomes of cohort 1 in the Edwards SAPIEN Aortic Bioprosthesis European Outcome (SOURCE) registry: the European registry of transcatheter aortic valve implantation using the Edwards SAPIEN valve. Circulation. 2011; 124(4): 425-433, doi:10.1161/CIRCULATIONAHA.110.001545, indexed in Pubmed: 21747054.

8. Moat NE, Ludman P, de Belder MA, et al. Long-term outcomes after transcatheter aortic valve implantation in high-risk patients with severe aortic stenosis: the U.K. TAVI (United Kingdom Transcatheter Aortic Valve Implantation) Registry. J Am Coll Cardiol. 2011; 58(20): 2130-2138, doi:10.1016/j.jacc.2011.08.050, indexed in Pubmed: 22019110.

9. Rodés-Cabau J, Webb JG, Cheung A, et al. Long-term outcomes after transcatheter aortic valve implantation: insights on prognostic factors and valve durability from the Canadian multicenter experience. J Am Coll Cardiol. 2012; 60(19): 1864-1875, doi: 10.1016/j.jacc.2012.08.960, indexed in Pubmed:23062535.

10. Hendel RC, Patel MR, Kramer CM, et al. American College of Cardiology Foundation Quality Strategic Directions Committee Appropriateness Criteria Working Group, American College of Radiology, Society of Cardiovascular Computed Tomography, Society for Cardiovascular Magnetic Resonance, American Society of Nuclear Cardiology, North American Society for Cardiac Imaging, Society for Cardiovascular Angiography and Interventions, Society of Interventional Radiology. ACCF/ACR/SCCT/SCMR/ ASNC/NASCI/SCAI/SIR 2006 appropriateness criteria for cardiac computed tomography and cardiac magnetic resonance imaging: a report of the American College of Cardiology Foundation Quality Strategic Directions Committee Appropriateness Criteria Working Group, American College of Radiology, Society of Cardiovascular Computed Tomography, Society for Cardiovascular Magnetic Resonance, American Society of Nuclear Cardiology, North American Society for Cardiac Imaging, Society for Cardiovascular Angiography and Interventions, and Society of Interventional Radiology. J Am Coll Cardiol. 2006; 48(7): 1475-1497, doi:10.1016/j. jacc.2006.07.003, indexed in Pubmed: 17010819.

11. Zamorano JL, Bax JJ, Rademakers FE. Evaluation of Systolic and Diastolic LV Function. The ESC Textbook of Cardiovascular Imaging. Springer, New York 2010: 307-320.

12. Buckert D, Dewes P, Walcher T, et al. Intermediate-term prognostic value of reversible perfusion deficit diagnosed by adenosine CMR: a prospective follow-up study in a consecutive patient population. JACC Cardiovasc Imaging. 2013; 6(1): 56-63, doi: 10.1016/j.jcmg.2012.08.011, indexed in Pubmed:23328562.

13. Buckert D, Kelle S, Buss S, et al. Left ventricular ejection fraction and presence of myocardial necrosis assessed by cardiac magnetic resonance imaging correctly risk stratify patients with stable coronary artery disease: a multi-center all-comers trial. Clin Res Cardiol. 2017; 106(3): 219-229, doi: 10.1007/s00392016-1042-5, indexed in Pubmed: 27738810.

14. Hombach V, Merkle N, Bernhard P, et al. Prognostic significance of cardiac magnetic resonance imaging: Update 2010. Cardiol J. 2010; 17(6): 549-557, indexed in Pubmed: 21154256.

15. Claus P, Omar AM, Pedrizzetti G, et al. Tissue tracking technology for assessing cardiac mechanics: principles, normal val- 
ues, and clinical applications. JACC Cardiovasc Imaging. 2015; 8(12): 1444-1460, doi: 10.1016/j.jcmg.2015.11.001, indexed in Pubmed: 26699113.

16. Pedrizzetti G, Claus P, Kilner PJ, et al. Principles of cardiovascular magnetic resonance feature tracking and echocardiographic speckle tracking for informed clinical use. J Cardiovasc Magn Reson. 2016; 18(1): 51, doi: 10.1186/s12968-016-0269-7, indexed in Pubmed: 27561421.

17. Pandis D, Sengupta PP, Castillo JG, et al. Assessment of longitudinal myocardial mechanics in patients with degenerative mitral valve regurgitation predicts postoperative worsening of left ventricular systolic function. J Am Soc Echocardiogr. 2014; 27(6): 627-638, doi: 10.1016/j.echo.2014.02.008, indexed in Pubmed: 24735653.

18. Reant P, Reynaud A, Pillois X, et al. Comparison of resting and exercise echocardiographic parameters as indicators of outcomes in hypertrophic cardiomyopathy. J Am Soc Echocardiogr. 2015; 28(2): 194-203, doi: 10.1016/j.echo.2014.10.001, indexed in Pubmed: 25459499.

19. Quarta CC, Solomon SD, Uraizee I, et al. Left ventricular structure and function in transthyretin-related versus light-chain cardiac amyloidosis. Circulation. 2014; 129(18): 1840-1849, doi: 10.1161/ CIRCULATIONAHA.113.006242, indexed in Pubmed: 24563469.

20. Nishimura RA, Otto CM, Bonow RO, et al. 2014 AHA/ACC guideline for the management of patients with valvular heart disease: executive summary: a report of the American College of Cardiology/American Heart Association Task Force on Practice Guidelines. J Am Coll Cardiol. 2014; 63: 2438-88.

21. Kramer CM, Barkhausen J, Flamm SD, et al. Society for Cardiovascular Magnetic Resonance Board of Trustees Task Force on Standardized Protocols. Standardized cardiovascular magnetic resonance (CMR) protocols 2013 update. J Cardiovasc Magn Reson. 2013; 15: 91, doi: 10.1186/1532-429X-15-91, indexed in Pubmed: 24103764.

22. Schulz-Menger J, Bluemke DA, Bremerich J, et al. Standardized image interpretation and post processing in cardiovascular magnetic resonance: Society for Cardiovascular Magnetic Resonance (SCMR) board of trustees task force on standardized post processing. J Cardiovasc Magn Reson. 2013; 15: 35, doi: 10.1186/1532-429X-15-35, indexed in Pubmed: 23634753.

23. Pedrizzetti G, Claus P, Kilner PJ, et al. Principles of cardiovascular magnetic resonance feature tracking and echocardiographic speckle tracking for informed clinical use. J Cardiovasc Magn Reson. 2016; 18(1): 51, doi: 10.1186/s12968-016-0269-7, indexed in Pubmed: 27561421.

24. Kappetein AP, Head SJ, Généreux P, et al. Updated standardized endpoint definitions for transcatheter aortic valve implantation: the Valve Academic Research Consortium-2 consensus document. EuroIntervention. 2012; 8(7): 782-795, doi: 10.4244/EIJV8I7A121, indexed in Pubmed: 23022744.

25. Thygesen K, Alpert JS, Jaffe AS, et al. Third universal definition of myocardial infarction. Eur Heart J. 2012; 33: 2551-67.

26. Anderson RP. First publications from the Society of Thoracic Surgeons National Database. Ann Thorac Surg. 1994; 57(1): 6-7, indexed in Pubmed:8279920.

27. Roques F, Nashef SA, Michel P, et al. Risk factors and outcome in European cardiac surgery: analysis of the EuroSCORE multinational database of 19030 patients. Eur J Cardiothorac Surg. 1999; 15(6): 816-22; discussion 822, indexed in Pubmed: 10431864.

28. Weidemann F, Jamal F, Sutherland GR, et al. Myocardial function defined by strain rate and strain during alterations in inotropic states and heart rate. Am J Physiol Heart Circ Physiol. 2002; 283(2): H792-H799, doi: 10.1152/ajpheart.00025.2002, indexed in Pubmed: 12124229.
29. Clemmensen TS, Eiskjær H, Løgstrup BB, et al. Left ventricular global longitudinal strain predicts major adverse cardiac events and all-cause mortality in heart transplant patients. J Heart Lung Transplant. 2017; 36(5): 567-576, doi: 10.1016/j. healun.2016.12.002, indexed in Pubmed:28089194.

30. Dahou A, Bartko PE, Capoulade R, et al. Usefulness of global left ventricular longitudinal strain for risk stratification in low ejection fraction, low-gradient aortic stenosis: results from the multicenter True or Pseudo-Severe Aortic Stenosis study. Circ Cardiovasc Imaging. 2015; 8(3): e002117, doi:10.1161/CIRCIMAGING.114.002117, indexed in Pubmed: 25681417.

31. Thavendiranathan P, Grant AD, Negishi T, et al. Reproducibility of echocardiographic techniques for sequential assessment of left ventricular ejection fraction and volumes: application to patients undergoing cancer chemotherapy. J Am Coll Cardiol. 2013; 61(1): 77-84, doi: 10.1016/j.jacc.2012.09.035, indexed in Pubmed: 23199515.

32. Macron L, Lairez O, Nahum J, et al. Impact of acoustic window on accuracy of longitudinal global strain: a comparison study to cardiac magnetic resonance. Eur J Echocardiogr. 2011; 12(5): 394-399, doi: 10.1093/ejechocard/jer029, indexed in Pubmed: 21471046.

33. Buss SJ, Breuninger K, Lehrke S, et al. Assessment of myocardial deformation with cardiac magnetic resonance strain imaging improves risk stratification in patients with dilated cardiomyopathy. Eur Heart J Cardiovasc Imaging. 2015; 16(3): 307-315, doi: 10.1093/ehjci/jeu181, indexed in Pubmed: 25246506.

34. Yang LiT, Yamashita E, Nagata Y, et al. Prognostic value of biventricular mechanical parameters assessed using cardiac magnetic resonance feature-tracking analysis to predict future cardiac events. J Magn Reson Imaging. 2017; 45(4): 1034-1045, doi: 10.1002/jmri.25433, indexed in Pubmed:27531830.

35. Delgado V, Tops LF, van Bommel RJ, et al. Strain analysis in patients with severe aortic stenosis and preserved left ventricular ejection fraction undergoing surgical valve replacement. Eur Heart J. 2009; 30(24): 3037-3047, doi: 10.1093/eurheartj/ehp351, indexed in Pubmed: 19726436.

36. Buckert D, Cieslik M, Tibi R, et al. Longitudinal strain assessed by cardiac magnetic resonance correlates to hemodynamic findings in patients with severe aortic stenosis and predicts positive remodeling after transcatheter aortic valve replacement. Clin Res Cardiol. 2017 [Epub ahead of print], doi: 10.1007/s00392017-1153-7, indexed in Pubmed: 28808772.

37. Dinh W, Nickl W, Smettan J, et al. Reduced global longitudinal strain in association to increased left ventricular mass in patients with aortic valve stenosis and normal ejection fraction: a hybrid study combining echocardiography and magnetic resonance imaging. Cardiovasc Ultrasound. 2010; 8: 29, doi: 10.1186/14767120-8-29, indexed in Pubmed: 20659321.

38. Bouleti C, Himbert D, Iung B, et al. Long-term outcome after transcatheter aortic valve implantation. Heart. 2015; 101(12): 936-942, doi:10.1136/heartjnl-2014-306694, indexed in Pubmed: 25655064.

39. Arnold SV, Afilalo J, Spertus JA, et al. Prediction of Poor Outcome After Transcatheter Aortic Valve Replacement. J Am Coll Cardiol. 2016; 68(17): 1868-1877, doi: 10.1016/j.jacc.2016.07.762, indexed in Pubmed: 27765189.

40. Reardon MJ, Van Mieghem NM, Popma JJ, et al. SURTAVI Investigators. Surgical or Transcatheter Aortic-Valve Replacement in Intermediate-Risk Patients. N Engl J Med. 2017; 376(14): 1321-1331, doi: 10.1056/NEJMoa1700456, indexed in Pubmed: 28304219 . 